\title{
RM22
}

\section{Use of Wavelets for Upscaling in Reservoir} Simulation

P.R. King* (Imperial College London) 
One of the recurrent problems in reservoir simulation is the range of length scales that have to be incorporated, from the scale of bedding planes (centimetres) to that of large scale flow units (100s of metres to kilometres). Incorporating all these scales either requires very fine grids (which would be inefficient) or some kind of localised grid refinement.

One approach to refining the grid is to use wavelet transforms. In this paper we use a Haar transform for single phase flow that effectively changes the pressure variables to the average and differences in pressures. With this transform, acting on the discretised flow equations, we can choose which parts of the problem we wish to retain. For example we can just ignore the (assumed) small differences or we can set a threshold on these errors. We find we can get a considerable improvement in compute speed without too great a loss of accuracy for a variety of problems. We then show how these ideas can be extended to simple multiphase problems. 